\title{
Transverse loop colostomy and colonic motility
}

\author{
F. Pucciani $\cdot$ M. N. Ringressi • \\ G. Maltinti $\cdot$ P. Bechi
}

Received: 13 April 2014/Accepted: 22 May 2014/Published online: 28 June 2014

(C) Springer-Verlag Italia Srl 2014

\begin{abstract}
Background The motility of the defunctionalized colon, distal to transverse loop colostomy, has never been studied "in vivo." The aim of our study was to evaluate the influence of transverse loop colostomy on colonic motility. Methods Thirteen patients were examined before stoma closure by means of clinical evaluation and colonic manometry; we studied both the right and distal colon in both fasting and fed patients in order to detect motor activity.

Results Quantitative and qualitative manometric analyses showed that the diverted colon had motor activity even if no regular colonic motor pattern was observed. The spreading of aboral propagated contractions (PCs) was sometimes recorded from the right colon to the distal colon. The response of the proximal and distal colon to a standard meal, when compared to fasting values, increased more than 40 and $35 \%$, respectively. Stool and gas ejections from the colostomy were never related to a particular type of colonic motility: Motor quiescence such as PCs was chaotically related to stool escape.

Conclusions In conclusion, motility of the defunctionalized colon is preserved in patients with transverse loop colostomy.
\end{abstract}

Keywords Transverse loop colostomy · Colonic motility $\cdot$ Colonic manometry $\cdot$ Low rectal resection

F. Pucciani $(\bowtie) \cdot$ M. N. Ringressi · G. Maltinti · P. Bechi Department of Surgery and Translational Medicine, University of Florence, Largo Brambilla 3, 50134 Florence, Italy e-mail: pucciani@unifi.it

\section{Introduction}

Temporary end or loop colostomy is carried out under various conditions: as an emergency procedure, in colorectal obstruction or perforation; as a protective measure, in low rectal resection and in the treatment of anastomotic leakage; to rest the colon affected by inflammatory bowel disease; and as a last resort in the treatment of severe fecal incontinence. The defunctionalized colon, distal to the colostomy, may be affected by diversion colitis [1], an atypical colitis that is thought to be due to the absence of the fecal stream since inflammatory changes spontaneously resolve following stomal closure [2]. Its etiology and pathogenesis are completely unknown [3]. Studies concerning the motility of the human defunctionalized colon are lacking. An experimental study on defunctionalized rat colon showed changes in nitrergic myenteric neurons, and the authors suggest that this report, by extrapolation, might explain the bowel dysmotility in humans after restoration of colonic continuity [4]. Another experimental study, performed on human colonic specimens, showed that the spontaneous motility and contractile response to electrical and pharmacological stimulation of the defunctionalized segments of the colon were similar to those of the nonexcluded colon [5]. Finally, a pediatric study of intractable childhood constipation associated with colonic distension showed that temporary diversion improved colonic motility; in this study, results of colonic manometry were used to predict which patients would benefit from resection or reanastomosis [6]. Since there are no "in vivo" studies of colonic motility in adults with temporary colostomy, the real motor pattern of the colon distal to the stoma is unknown.

It is well accepted that transverse loop colostomy or ileostomy has a protective role in terms of consequences of 
anastomotic dehiscence after anterior resection of the rectum [7]. More specifically, its construction seems mandatory when an ultralow anterior resection is performed [8]. Although it is not clear which diverting stoma is preferable, both stomas might produce a doubly favorable effect on the anastomosis. The first diverts the fecal stream, and the second can lessen downstream colonic motility. We undertook the present study in order to investigate this second possibility in patients with transverse loop colostomy.

The primary endpoint was detection of motility in colonic sections proximally and distally to transverse colostomy; the secondary endpoint was identification of motility patterns of the defunctionalized colon.

\section{Materials and methods}

Between February 2010 and April 2013, 16 patients with rectal cancer underwent temporary transverse loop colostomy. Colostomy was performed as a protective measure in low anterior rectal resection procedures and as treatment for anastomotic leakage in six of the patients. After exclusion of three patients due to a history of irritable bowel syndrome, the remaining 13 (5 men and 8 women; mean age 61 years, range $48-82$ years), without endoscopic evidence of diversion colitis, were enrolled in the study. All 13 patients were examined before stoma closure time: 5 at 2 months after colostomy and 8 at 2 months after the end of adjuvant therapy. All underwent clinical examination and colonic manometry; previous oncological evaluation excluded recurrence and/or metastatic disease and a complete colonoscopy excluded organic causes that might influence colonic motility.

According to the ethics guidelines of our university consistent with the Helsinki declaration, all patients provided written informed consent.

\section{Clinical evaluation}

All patients underwent clinical evaluation. Information regarding bowel movements per $24 \mathrm{~h}$ was reported; the number of stoma bags/day was recorded, and stool form was classified according to the Bristol scale [9]. The following stoma-related complications were noted: parastomal hernia, prolapse, stenosis, peristomal dermatitis, and mucosal edema.

\section{Colonic manometry}

Stationary colonic manometry was conducted according to the American Neurogastroenterology and Motility Society consensus statement [10]. Colonic manometry was performed using a stationary laboratory-based computerized system (Dyno Compact, Menfis Division-Medica s.p.a., Modena, Italy). Laxatives and drugs affecting intestinal motility were discontinued at least 3 days before the study. The day before the study, the descending colon, efferent from colostomy, was cleaned by an enema. The study was carried out by the same physician (FP) after patients had fasted for $12 \mathrm{~h}$. Two 4-channel catheters (EUCAT, Medimar s.r.l., Milan, Italy) with 4 holes $5 \mathrm{~cm}$ apart from each other, perfused at $0.5 \mathrm{ml} / \mathrm{min}$ by a lowcompliance perfusion pump, were used. The first catheter was placed with the distal recording site located at $25 \mathrm{~cm}$ in the afferent upstream of the transverse colostomy; manometric recordings of ascending colon and proximal transverse colon were then performed. The second catheter was placed with the distal recording site located at $25 \mathrm{~cm}$ in the efferent downstream of the transverse colostomy; in this way, colonic manometry recorded motility in the distal colon. The catheter position was confirmed with brief fluoroscopy. Motility was recorded in all fasting patients for $4 \mathrm{~h}$ and then in fed patients (after a typical Italian meal of 950 calories including bread, pasta with sauce, chicken, potatoes, and fresh fruit) for an additional $2 \mathrm{~h}$. Manometric recordings were evaluated separately in the fasting and fed states in each of the recording sites. (1) Qualitative analysis. Algorithms for computerized analysis were in accordance with validated parameters suggested by De Schryver et al. [11]. Computerized algorithms identified common colonic motor patterns such as propagated contractions (PC), retrograde contractions (RC), simultaneous contractions (SC), and high-amplitude propagated contractions (HAPC). Propagated contractions were aborad migrating waves across $>10 \mathrm{~cm}$ at a velocity $>0.5 \mathrm{~cm} \mathrm{~s}^{-1}$. RCs migrated orad across $>15 \mathrm{~cm}$ with a velocity $>0.5 \mathrm{~cm} \mathrm{~s}^{-1}$. SCs occurred simultaneously at least $10 \mathrm{~cm}$ apart. HAPC were pressure waves $>75 \mathrm{mmHg}$ that migrated aborad for $>15 \mathrm{~cm}$. (2) Quantitative analysis. Phasic pressure activity was summarized as motility index (MI) $\left[\log _{\mathrm{e}}(\mathrm{sum}\right.$ of amplitudes $\times$ number of contractions +1$)]$; mean amplitude $(\mathrm{mmHg})$ and propagation velocity $(\mathrm{mm} / \mathrm{sec})$ of pressure waves were noted [10].

Statistical analysis

Sample size adequacy and statistical power of the study were calculated (DSS Research, statistical power calculator). The results are expressed as the mean \pm standard deviation (SD). Student's $t$ test for paired and unpaired samples was used for statistical analyses. All correlations were evaluated using Spearman's rank correlation coefficient $\left(\rho_{\mathrm{s}}\right)$. A $p$ value $<0.05$ was chosen for rejection of the null hypothesis. 


\section{Results}

The sample size was adequate (adequacy $\pi=0.80$ ) to achieve a statistical power of $100 \%$. The mean time from surgery to final evaluation was 5.7 (range 2-9) months. Clinical evaluation is reported in Table 1. Most patients' stool was of normal consistency. Four patients $(30.7 \%)$ had loose stool. The number of stoma bags used per day was usually low, but the number was increased $(3.3 \pm 0.56)$ when loose stool was predominant. Three patients had peristomal dermatitis and one had signs of parastomal hernia. The results of colonic manometry are shown in Table 2. No regular motility pattern was observed. Periods of motor quiescence alternated with absolutely chaotic motor activity in both the fasting and fed states. Stool and gas ejection from the colostomy occurred in every subject, more often after a meal than before. No specific motor pattern was associated with gas or stool emission (Table 3). Isolated propagated waves or HAPC were more often recorded before stool ejection (Fig. 1), and a significant correlation was found $\left(\rho_{\mathrm{s}}=0.74\right.$; $p<0.03)$. Nevertheless, motor quiescence was sometimes $(16.6 \%)$ observed during stool movements. During fasting, colonic motility parameters were quite low, with significantly lower values in the colon distal to the colostomy

Table 1 Clinical evaluation

\begin{tabular}{ll}
\hline Stool form & \\
Hard stool (Bristol scale 1 or 2) & $0 / 13$ \\
Normal stool consistency (Bristol scale 3, 4, or 5) & $4 / 13$ \\
Loose stool (Bristol scale 6 or 7) & $9 / 13$ \\
Stoma bags ( $n$ /day) & $1.84 \pm 0.35$ \\
Parastomal hernia ( $n$ patients/total patients) & 1 \\
Prolapse ( $n$ patients/total patients) & 0 \\
Peristomal dermatitis ( $n$ patients/total patients) & 3 \\
Mucosal edema & 2 \\
\hline
\end{tabular}

when compared to those in the colon proximal to the colostomy (Table 3). Particularly, motility indexes showed significant differences between the two recording sites $(p<0.002)$ (Fig. 2). All patients, except for $2(15.3 \%)$, had a colonic motility pattern in which motility increased significantly after a meal $(p<0.01)$ and was maintained throughout the entire post-feeding period. These two patients had a lower fasting colonic motility pattern after eating: the MI shifted from $261 \pm 43$ (pre-meal) to $187 \pm 31$ (post-meal). However, the distal colon exhibited significantly less MI than the proximal colon $(p<0.002)$ in all patients. The propagation velocity of pressure waves did not show significant differences between colonic segments. Interestingly, the propagation of pressure waves could sometimes be recorded from segments proximal to the colostomy toward the distal segments (Fig. 3); 11 proximal PC (37.9\%) and 13 proximal HAPC (72.2 \%) spread distally. Three times even RC propagated orally through the colostomy.

\section{Discussion}

Endoscopic evidence of disuse colitis can be found in $70-90 \%$ of patients with fecal diversion [2, 12], and lymphoid follicular hyperplasia is described as a distinctive

Table 3 Relationship between number of stool-gas ejections and colonic contractions

\begin{tabular}{lllr}
\hline & \multicolumn{2}{l}{ Stool } & Gas \\
\cline { 2 - 3 } & Solid & Loose & \\
\hline Propagated contractions & 2 & 8 & 19 \\
Simultaneous contractions & 0 & 1 & 2 \\
Retrograde contractions & 0 & 0 & 1 \\
High-amplitude propagated contractions & 4 & 5 & 9 \\
Motor quiescence & 0 & 4 & 4 \\
\hline
\end{tabular}

Table 2 Colonic manometry

\footnotetext{
${ }^{\circ}$ After meal-up to colostomy versus down from colostomy: $p<0.001 . *$ Up to colostomyafter meal versus fasting: $p<0.002$. ** Down from colostomy: after meal versus fasting: $p<0.03{ }^{\circ}$ Fasting —up to colostomy versus down from colostomy: $p<0.02$
}

\begin{tabular}{|c|c|c|c|c|}
\hline & \multicolumn{2}{|l|}{ Fasting } & \multicolumn{2}{|l|}{ After meal } \\
\hline & $\begin{array}{l}\text { Up to } \\
\text { colostomy }\end{array}$ & $\begin{array}{l}\text { Down from } \\
\text { colostomy }\end{array}$ & Up to colostomy & $\begin{array}{l}\text { Down from } \\
\text { colostomy }\end{array}$ \\
\hline Number of pressure waves & $99.7 \pm 22.8^{\circ}$ & $46.5 \pm 19.4$ & $203.5 \pm 26.4 * \circ$ & $92.3 \pm 23.8^{* *}$ \\
\hline $\begin{array}{l}\text { Mean amplitude pressure waves } \\
(\mathrm{mmHg})\end{array}$ & $21.8 \pm 5.7$ & $21.2 \pm 7.9$ & $32.0 \pm 13.6$ & $24.2 \pm 7.0$ \\
\hline Velocity propagation $(\mathrm{mm} / \mathrm{sec})$ & $32.6 \pm 9.5$ & $24.4 \pm 4.5$ & $25.6 \pm 9.8$ & $28.7 \pm 8.4$ \\
\hline Propagated contractions $(n)$ & $6.5 \pm 1.7^{\circ}$ & $2.5 \pm 1.8$ & $17.0 \pm 4.4 * \circ$ & $3.2 \pm 2.2$ \\
\hline Retrograde contractions $(n)$ & $4.7 \pm 2.8$ & $3.1 \pm 2.8$ & $6.7 \pm 4.9$ & $4.3 \pm 3.5$ \\
\hline Simultaneous contractions $(n)$ & $2.5 \pm 1.5$ & $1.25 \pm 0.15$ & $2.75 \pm 1.75$ & $1.75 \pm 0.45$ \\
\hline $\begin{array}{l}\text { High-amplitude propagated } \\
\text { contractions }(n)\end{array}$ & $0.8 \pm 0.35$ & $0.75 \pm 0.45$ & $1.75 \pm 0.25$ & $1.4 \pm 0.5$ \\
\hline
\end{tabular}




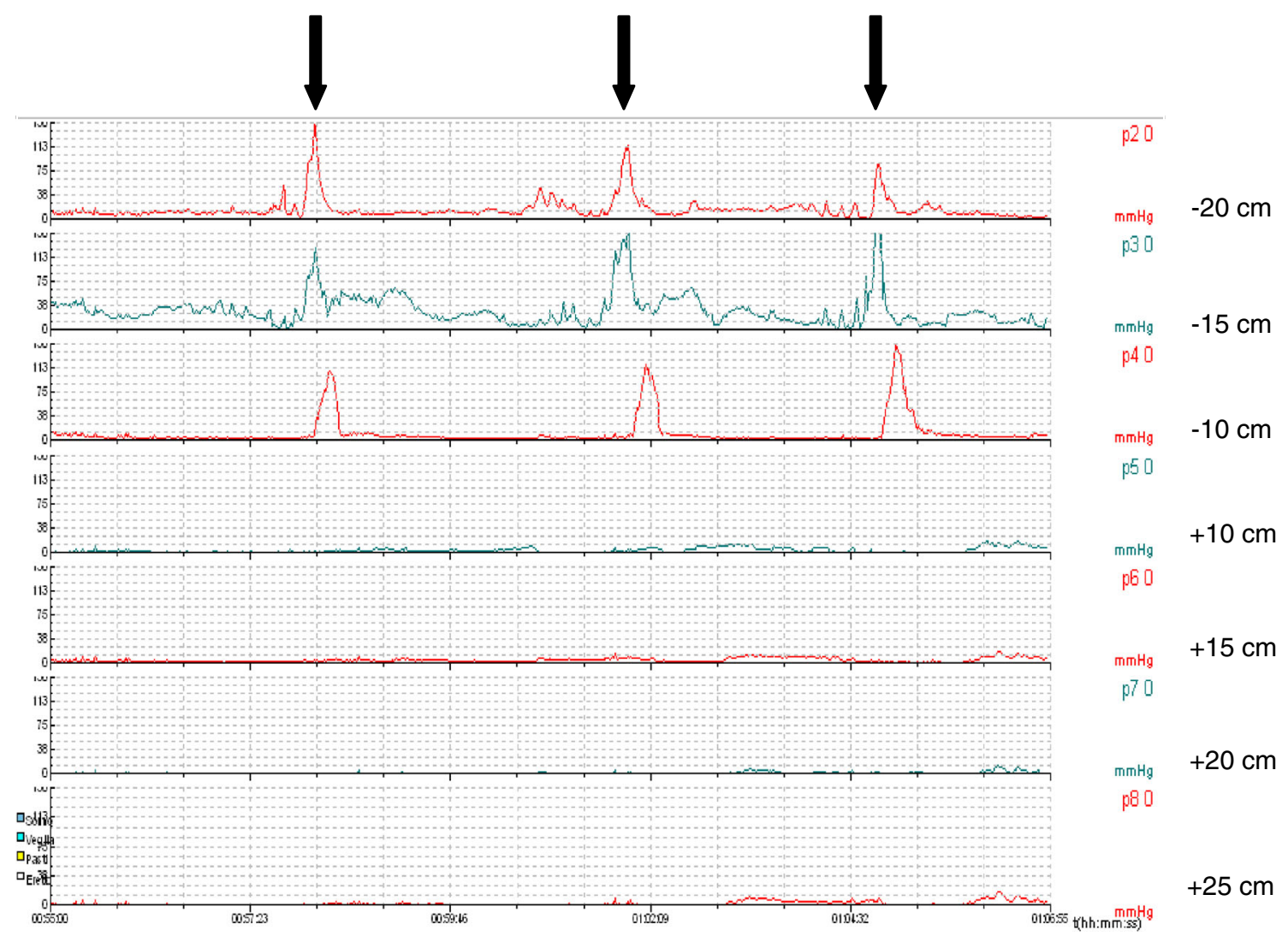

Fig. 1 Colonic motility. Manometric recordings during stool ejection (down arrow). Note manometric waves in recording sites proximal to the colostomy $(-20,-15,-10 \mathrm{~cm})$ and manometric silence in recording sites distal to the colostomy $(+10,+15,+20,+25 \mathrm{~cm})$

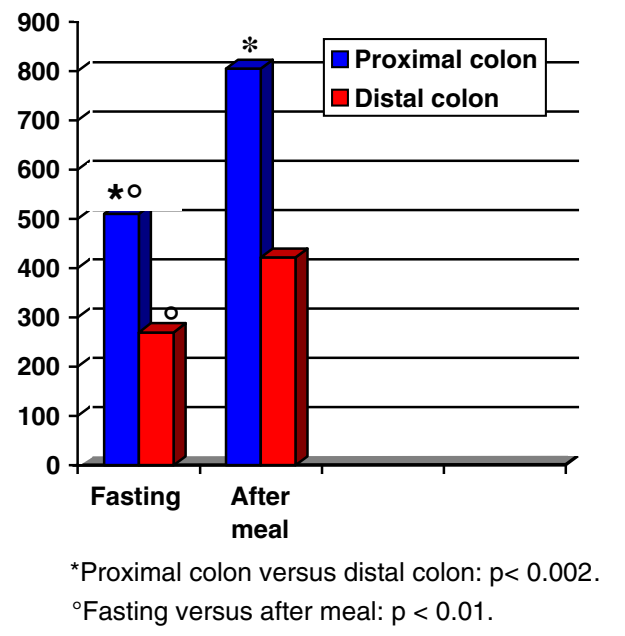

Fig. 2 Fasting and after meal motility indexes in colonic segments, proximal and distal to the colostomy

pathological finding independently of the condition of the colon before diversion [13]. The constant resolution of diversion colitis following stomal closure demonstrates that restoring the fecal stream cures diversion colitis $[1,3]$. The therapeutic success of short-chain fatty acid enema irrigation with high-dose butyric acid, a colonic nutrient that is in the fecal stream, supports the relevance of stool transit to colonic trophism [14]. Few papers focus on motility of the diverted colon, and there are no published reports with "in vivo" findings. Our research was carried out by means of colonic manometric recordings through transverse loop colostomy: We could study the colon, before and after stoma, in order to observe the influence of colostomy on colonic motility. After low anterior resection, the sigmoid colon and rectum are removed and the transverse and descending colon are mobilized to make the colorectal or coloanal anastomosis. In this way, motility recordings at $25 \mathrm{~cm}$ distal to the transverse colostomy allow detection of colonic motility for a relatively long tract of distal residual colon. Colonic manometry was performed according to the protocol suggested by the American Neurogastroenterology and Motility Society [10]: Recording time (conducted for $6 \mathrm{~h}$ ) and pattern detection of colonic phasic pressure activity (PC, RC, SC, HAPC, and MI) were those suggested for stationary laboratory-based studies for assessing colonic motility. Evaluation of our colonic tracings was limited to their absolute analyses and to a comparison of findings proximal and distal to colostomy. We did not attempt to compare our 


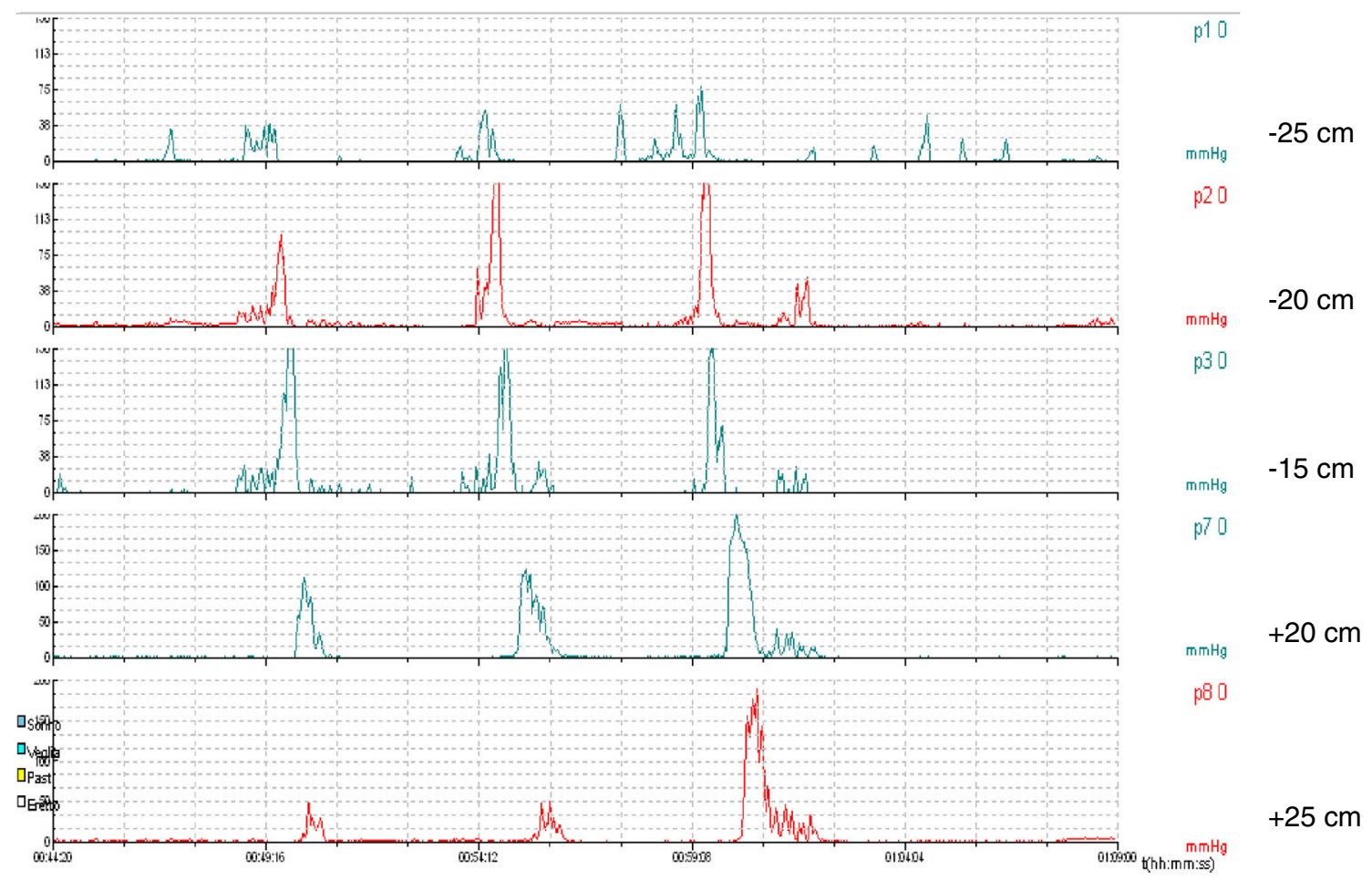

Fig. 3 Propagated motor activity at 25, 20, and $15 \mathrm{~cm}$ upstream to the colostomy and at 20 and $25 \mathrm{~cm}$ downstream from the colostomy

patients with normal subjects since internationally acknowledged normal manometric values are lacking. We found that fasting and fed manometric recordings show that both pre-stomal and post-stomal colonic segments have similar motility patterns with motor quiescence alternating with motor dynamics, although there is less motor activity in the distal colon than in the proximal colon. The latter finding is consistent with previous reports in healthy subjects $[15,16]$ and, therefore, seems to confirm that there are no significant changes in motor activity of the diverted colon, in agreement with what was suggested in the previously cited study on colonic specimens [5]. More specifically, quantitative analysis shows that the response of the proximal and distal colon to the meal, when compared to fasting values, increases more than 40 and $35 \%$, respectively, in line with a normal motor response of the intact colon to food consumption [10]. We cannot explain the lack of greater motility in two patients: Perhaps, the colonic response to eating would have been triggered by consumption of more than 950 calories. The integrity of the motor activity pattern is, however, also confirmed by evidence of the spreading of aboral propagated contractions from the right colon to the distal colon (Fig. 3). Therefore, transverse loop colostomy does not impair neuroendocrine connections of the distal colon: probably, thanks to careful preservation of the mesocolon. Last but not least, it is not possible to identify, by means of colonic manometry, a precise relationship between stool ejection from colostomy and any particular colonic motor pattern. Stool ejection occurs chaotically, often alternating between solid and loose stools. In the same way, stool leakage does not seem to be related to specific motor activity; usually, during stool escape, high-amplitude propagated contractions and isolated contractions succeed one another, but sometimes motor quiescence may be recorded.

\section{Conclusions}

Colonic motility is maintained in the downstream colon after transverse loop colostomy. Therefore, the only beneficial effect which can be expected from colostomy when performed in low rectal resection is the exclusion of the colorectal anastomosis from the fecal stream. On the contrary, no favorable effect due to decreased motility can be expected. Overall, the question regarding motor behavior of the diverted colon after stomal closure and fecal stream restoration is still unanswered. Possible clinical motility disorders after colorectal restoration might emerge, perhaps due to removed sigmoid colon rather than smooth muscle structural or functional changes related to previous defunctionalization [5]; it is known that sphincter-saving resections may promote a faster colonic transit time by removing sigmoid segmental activity [17]. However, 
further colonic motor studies are necessary to understand bowel dysmotility that could appear after restoration of colonic continuity.

\section{Conflict of interest None.}

\section{References}

1. Thorsen AJ (2007) Non-infectious colitides: collagenous colitis, lymphocytic colitis, diversion colitis and chemically induced colitis. Clin Colon Rectal Surg 20:47-57

2. Whelan RL, Abramson D, Kim DS, Hashmi HF (1994) Diversion colitis. A prospective study. Surg Endosc 8:19-24

3. Kabir SA, Kabir SI, Richards ER, Ahmed J, McNaught C (2012) Diversion colitis: the mystery remains. Br J Surg 99-S6:S81

4. Chaudury A, Shariff A, Srinivas M, Sabherwal U (2004) Changes in nitrergic innervation of defunctionalized rat colon after diversion colostomy. Neurogastroenterol Motil 16:475-487

5. Violi V, Cobianchi F, Adami M, Torri T, Ferraro G, Roncoroni L (1998) Human defunctionalized colon. A histopathological and pharmacological study of muscularis propria in resection specimens. Dig Dis Sci 43:616-623

6. Villareal J, Sood M, Zangen T et al (2001) Colonic diversion for intractable constipation in children: colonic manometry helps guide clinical decisions. J Pediatr Gastroenterol Nutr 33:588-591

7. Marusch F, Koch A, Schmidt U et al (2002) Value of a protective stoma in low anterior resections for rectal cancer. Dis Colon Rectum 45:1164-1171
8. Tjandra JJ, Chan MK, Yeh CH (2008) Laparoscopic-versus handassisted ultralow anterior resection: a prospective study. Dis Colon Rectum 51:26-31

9. Heaton KW, Radvan J, Cripps H, Mountford RA, Braddon FEM, Hughes A (1992) Defecation frequency and timing and stool form in the general population: a prospective study. Gut 33:818-824

10. Camilleri M, Bharucha AE, Di Lorenzo C et al (2008) American Neurogastroenterology and Motility Society consensus statement on intraluminal measurement of gastrointestinal and colonic motility in clinical practice. Neurogastroenterol Motil 20:1269-1282

11. De Schryver AMP, Samsom M, Akkermans LMA, Clemens CHM, Smout AJPM (2002) Fully automated analysis for colonic manometry recordings. Neurogastroenterol Motil 14:697-703

12. Ferguson CM, Siegel RJ (1991) A prospective evaluation of diversion colitis. Am Surg 57:46-49

13. Yeong ML, Bethwaite PB, Prasad J, Isbister WH (1991) Lymphoid follicular hyperplasia: a distinctive feature of diversion colitis. Histopathology 19:55-61

14. Edwards CM, George B, Warren BF (1999) Diversion colitis: new light through old windows. Histopathology 34:1-5

15. Bassotti G, Imbimbo BP, Gaburri M, Daniotti S (1987) Transverse and sigmoid colon motility in healthy humans: effects of eating and cimetropium bromide. Digestion 37:59-64

16. Hagger A, Kumar D, Benson M, Grundy A (2002) Periodic colonic motor activity identified by 24-h pancolonic ambulatory manometry in humans. Neurogastroenterol Motil 14:271-278

17. Bassotti G, de Roberto G, Chistolini F, Morelli A, Pucciani F (2005) Case report: colonic manometry reveals abnormal propulsive behaviour after anterior resection of the rectum. Dig Liver Dis 37:124-128 